HOTEL INTERCONTINENTAL HOTEL INTERCONTINENTAL HOTEL INTERCONTI NENTAL HOTEL INTERCONTINENTAL HOTEL INTERCONTINENTAL HOTEL IN TERCONTINENTAL HOTEL INTERCONTINENTAL HOTEL INTERCONTINENTAL HOTEL INTERCONTINENTAL HOTEL INTERCONTINENTAL HOTEL INTERCONTI NENTAL HOTEL INTERCONTINENTAL HOTEL INTERCONTINENTAL HOTEL IN TERCONTINENTAL HOTEL INTERCONTINENTAL HOTEL INTERCONTINENTAL HOTEL INTERCONTINENTAL HOTEL INTERCONTINENTAL HOTEL INTERCONTI NENTAL HOTEL INTERCONTINENTAL HOTEL INTERCONTINENTAL HOTEL IN TERCONTINENTAL HOTEL INTERCONTINENTAL HOTEL INTERCONTINENTAL HOTEL INTERCONTINENTAL HOTEL INTERCONTINENTAL HOTEL INTERCONTI NENTAL HOTEL INTERCONTINENTAL HOTEL INTERCONTINENTAL HOTEL IN TERCONTINENTAL HOTEL INTERCONTINENTAL HOTEL INTERCONTINENTAL HOTEL INTERCONTINENTAL HOTEL INTERCONTINENTAL HOTEL INTERCONTI NENTAL HOTEL INTERCONTINENTAL HOTEL INTERCONTINENTAL HOTEL IN TERCONTINENTAL HOTEL INTERCONTINENTAL HOTEL INTERCONTINENTAL HOTEL INTERCONTINENTAL HOTEL INTERCONTINENTAL HOTEL INTERCONTI NENTAL HOTEL INTERCONTINENTAL HOTEL INTERCONTINENTAL HOTEL IN TERCONTINENTAL HOTEL IN HOTEL INTERCONTINENTAL ト NENTAL HOTEL INTERCONTII TERCONTINENTAL HOTEL IN HOTEL INTERCONTINENTAL \& NENTAL HOTEL INTERCONTII TERCONTINENTAL HOTEL IN HOTEL INTERCONTINENTAL \& NENTAL HOTEL INTERCONTII TERCONTIN

HOTEL INTI

NENTAL HC TERCONTIN HOTEL INTE NENTAL HC TERCONTIN HOTEL INTI NENTAL HC TERCONTINENTAL HOTEL IN HOTEL INTERCONTINENTAL \& NENTAL HOTEL INTERCONTII TERCONTINENTAL HOTEL IN HOTEL INTERCONTINENTAL + NENTAL HOTEL INTERCONTII TERCONTINENTAL HOTEL IN HOTEL INTERCONTINENTAL \& NENTAL HOTEL INTERCONTII I HOTEL INTERCONTINENTAL INENTAL HOTEL INTERCONTI ITERCONTINENTAL HOTEL IN I HOTEL INTERCONTINENTAL INENTAL HOTEL INTERCONTI ITERCONTINENTAL HOTEL IN I HOTEL INTERCONTINENTAL INENTAL HOTEL INTERCONTI ITERCONTINENTAL HOTEL IN NTINENTAL NTERCONTI 1 HOTEL IN GENÈVE NTINENTAL NTERCONTI I HOTEL IN NTINENTAL NTERCONTI I HOTEL IN I HOTEL INTERCONTINENTAL INENTAL HOTEL INTERCONTI ITERCONTINENTAL HOTEL IN I HOTEL INTERCONTINENTAL INENTAL HOTEL. INTERCONTI ITERCONTINENTAL HOTEL IN I HOTEL INTERCONTINENTAL INENTAL HOTEL INTERCONTI ITERCONTINENTAL HOTEL IN TERCONTINENTAL HOTEL INTERCONTINENTAL HOTEL INTERCONTINENTAL HOTEL INTERCONTINENTAL HOTEL INTERCONTINENTAL HOTEL INTERCONTI NENTAL HOTEL INTERCONTINENTAL HOTEL INTERCONTINENTAL HOTEL IN TERCONTINENTAL HOTEL INTERCONTINENTAL HOTEL INTERCONTINENTAL HOTEL INTERCONTINENTAL HOTEL INTERCONTINENTAL HOTEL INTERCONTI NENTAL HOTEL INTERCONTINENTAL HOTEL INTERCONTINENTAL HOTEL IN TERCONTINENTAL HOTEL INTERCONTINENTAL HOTEL INTERCONTINENTAL HOTEL INTERCONTINENTAL HOTEL INTERCONTINENTAL HOTEL INTERCONTI NENTAL HOTEL INTERCONTINENTAL HOTEL INTERCONTINENTAL HOTEL IN TERCONTINENTAL HOTEL INTERCONTINENTAL HOTEL INTERCONTINENTAL HOTEL INTERCONTINENTAL HOTEL INTERCONTINENTAL HOTEL INTERCONTI NENTAL HOTEL INTERCONTINENTAL HOTEL INTERCONTINENTAL HOTEL IN TERCONTINENTAL HOTEL INTERCONTINENTAL HOTEL INTERCONTINENTAL HOTEL INTERCONTINENTAL HOTEL INTERCONTINENTAL HOTEL INTERCONTI NENTAL HOTEL INTERCONTINENTAL HOTEL INTERCONTINENTAL HOTEL IN TERCONTINENTAL HOTEL INTERCONTINENTAL HOTEL INTERCONTINENTAL HOTEL INTERCONTINENTAL HOTEL INTERCONTINENTAL HOTEL INTERCONTI NENTAL HOTEL INTERCONTINENTAL HOTEL INTERCONTINENTAL HOTEL IN TERCONTINENTAL HOTEL INTERCONTINENTAL HOTEL INTERCONTINENTAL HOTEL INTERCONTINENTAL HOTEL INTERCONTINENTAL HOTEL INTERCONTI 


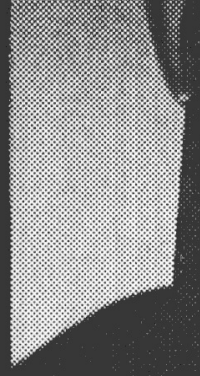

kx 


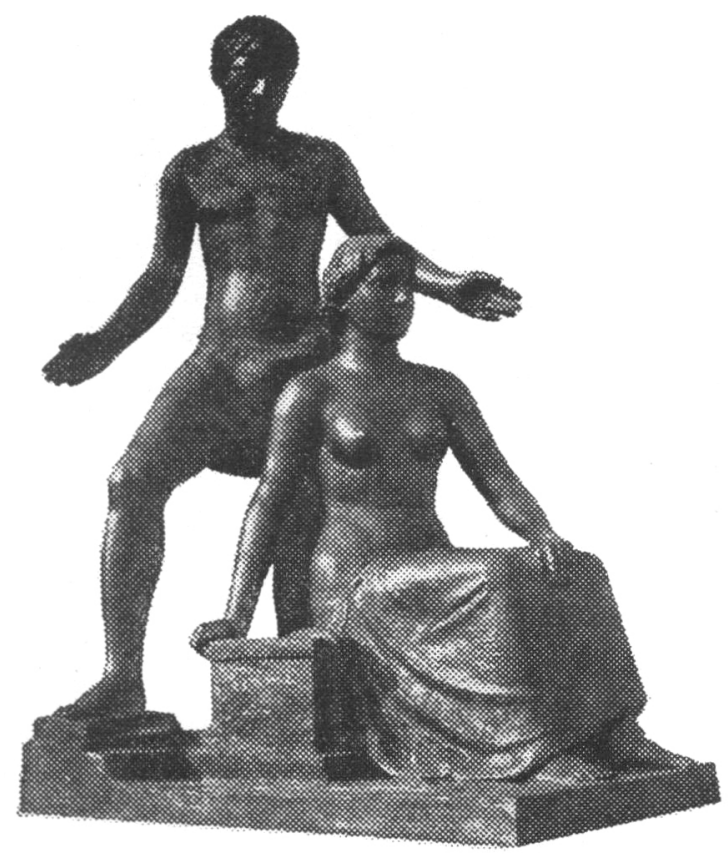

Société suisse

d'Assurances générales sur la vie humaine

RENTENANSTALT

Entreprise mutuelle

Fondée en 1857

Siège à Zurich $2^{\mathrm{e}}, 40$, quai du Général-Guisan

Agences générales à :

Aarau, Bále, Berne, Bienne, Coire, Fribourg, Genèe, Glaris, Lausanne, Lucerne,

Lugano, Neuchatel, Romanshorn, Saint-Gall, Sion, Soleure, Winterthour, Zurich

Sièges spéciaux à :

Munich - Paris - Amsterdan - Bruxelles

La plus ancienne et la plus grande des soclétés suisses d'assurances sur la vie 

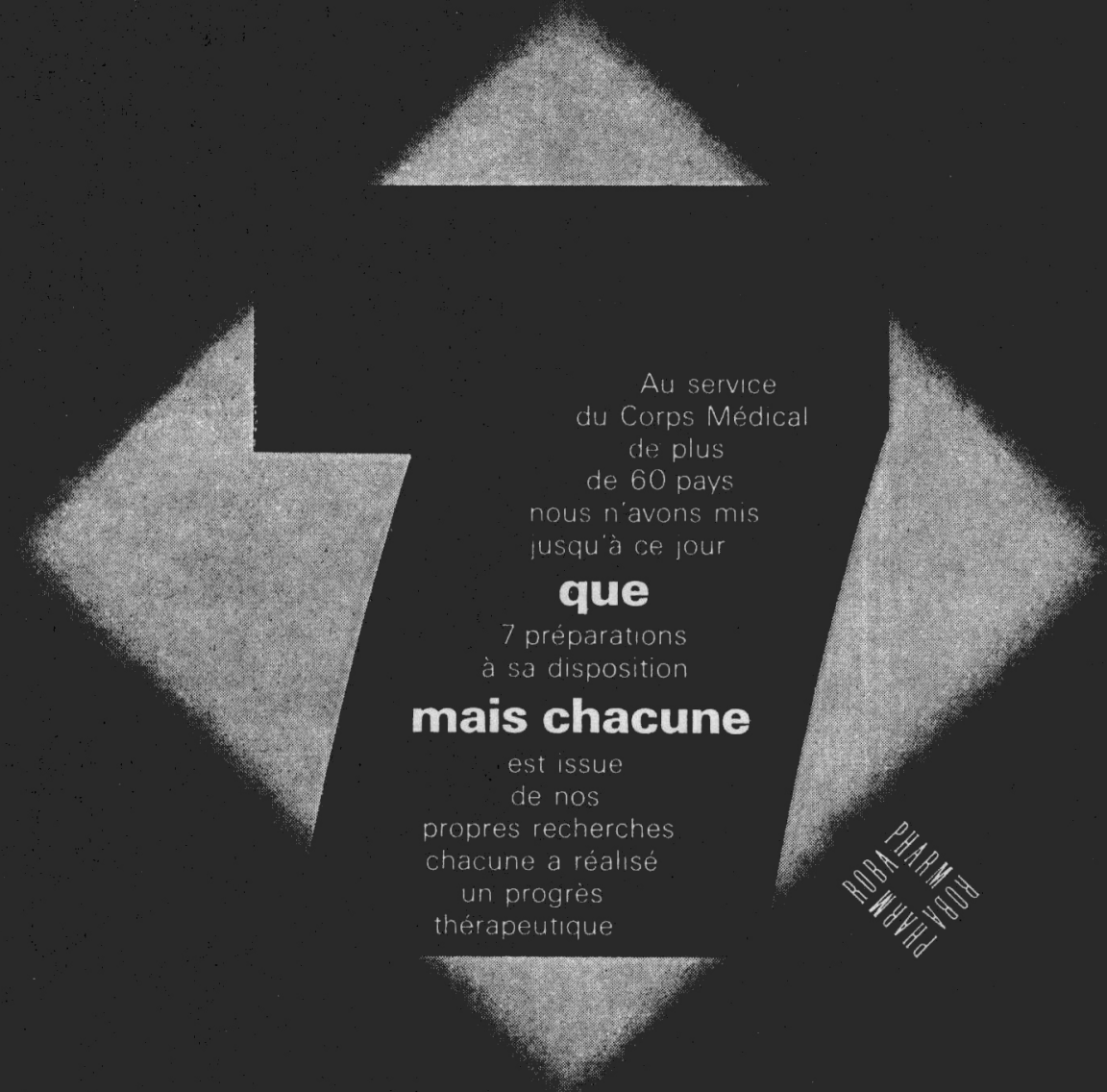

\section{Robapharm Bâle}




\section{Pas d'hésitation sur la voie à suivre}

Une étape importante dans l'étude de nouveaux produits : parvenir à fixer des substances marquées et leurs métabolites radioactifs sur les organes et les tissus. L'autoradiographie de l'animal intact apporte alors une moisson d'informations.

Lorsqu'il est nécessaire de suivre cette voie pour étudier un médicament ou de perfectionner au maximum des méthodes d'investigation, aucun sacrifice n'est trop grand pour Bayer.

\section{0 millions de marks pour notre nouveau centre de recherche}
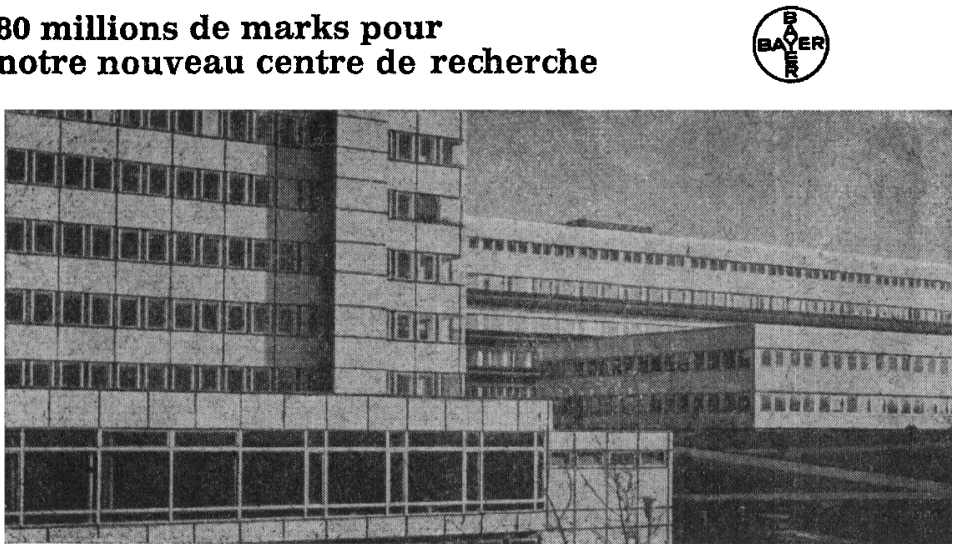

Mettre à la disposition des chercheurs scientifiques des techniques de travail parfaites est indispensable pour la création et l'expérimentation rigoureuse de nouveaux produits.

Pas d'hésitation sur la voie à suivre:

Conserver la confiance des médecins et des. malades en leur offrant des médicaments toujours meilleurs. 


\section{Laboratoires \\ VIFOR S.A.}

\section{GENEVE}

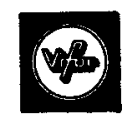

\section{Spécialités pharmaceutiques}

\section{MOSER \& SAUVAIN}

Décoration d'intérieurs - Moquettes - Tentures - Rideaux

Papiers peints - Revêtements de sols 


\section{TRPMARSP}

- transports aériens

Agents maritimes

GENEVVE, 17, rue du Mont-Blanc

Succursales et agences: Bâle, Le Locle, Sion, Zurich

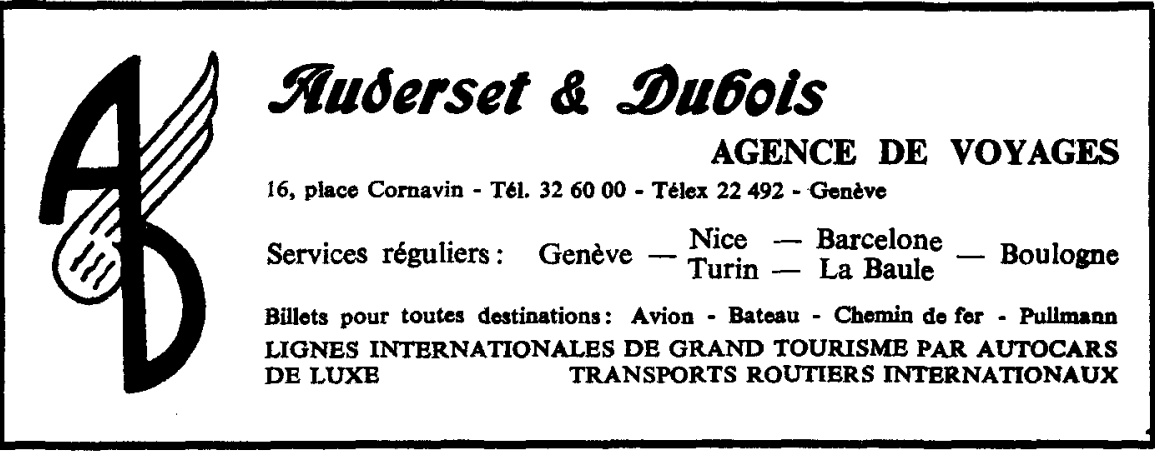

\section{LOUIS DUPONT}

Entreprises sanitaires

Ferblanterie el Couverture

G E N È V E

Tél. 243283 


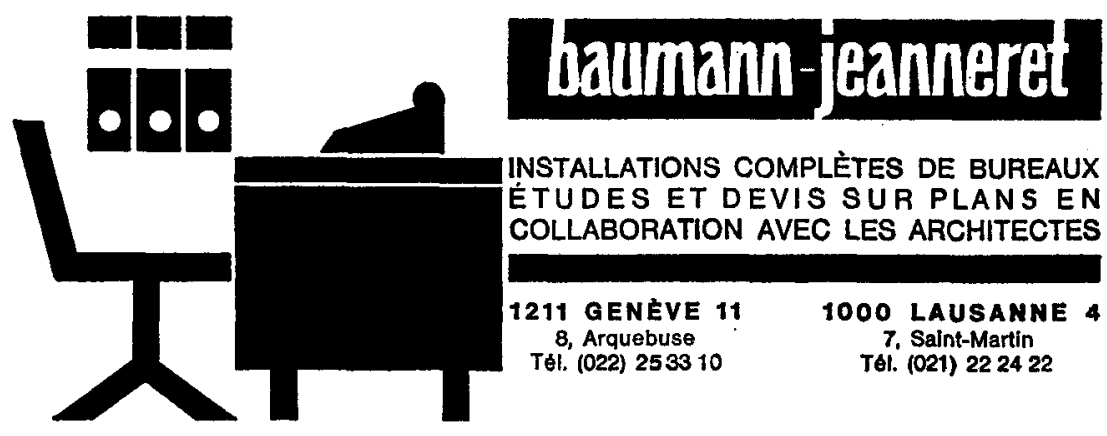

Montex S.a. 4.roo ou montrature

Exportation - Importation Genève

Téléphone: (022) 320012

Tólex 23458

Cables : Corvin Geneve
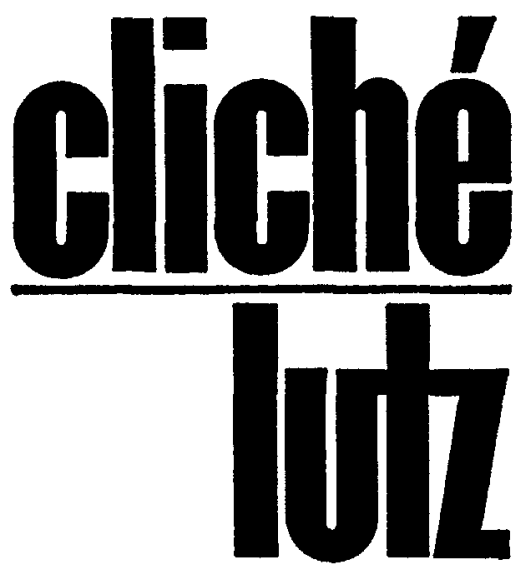

Zurich $\varnothing 051 / 424211$

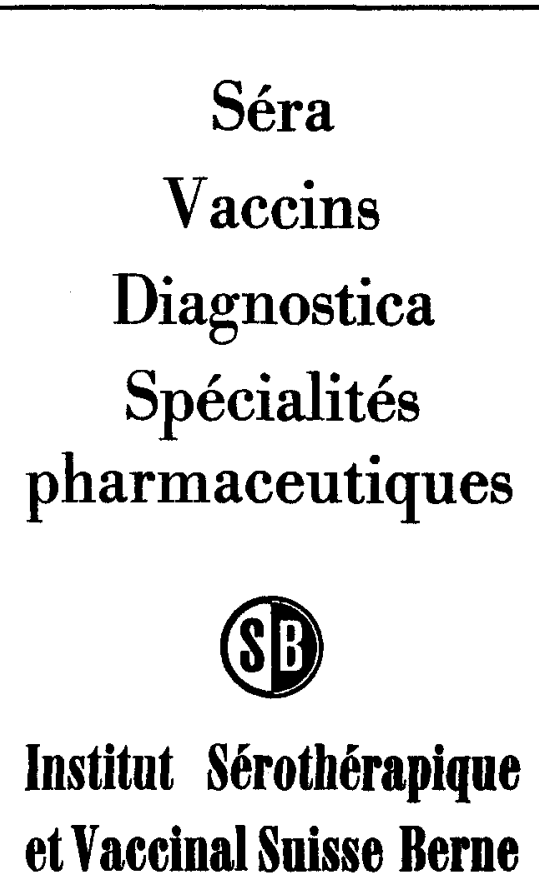




\section{EXTRAIT DES STATUTS \\ DU COMITE INTERNATIONAL DE LA CROIX-ROUGE (adoptés le 25 septembre I952)}

ARticle PRemier. - Le Comité international de la Croix-Rouge (CICR), fondé à Genève en r863, consacré par les Conventions de Genève et par les Conférences internationales de la Croix-Rouge, est une institution indépendante ayant son statut propre.

Il est partie constitutive de la Croix-Rouge internationale 1.

ART. 2 - En tant qu'association régie par les articles 60 et suivants du Code civil suisse, le CICR possède la personnalité civile.

ART. 3. - Le CICR a son siège à Genève.

Il a pour emblème la croix rouge sur fond blanc. Sa devise est «Inter arma caritas ».

ART. 4. - Le CICR a, notamment, pour rôle :

a) de maintenir les principes fondamentaux et permanents de la CroixRouge, à savoir: l'impartialité, une action indépendante de toute considération raciale, politique, confessionnelle ou économique, l'universalité de la Croix-Rouge et l'égalité des Sociétés nationales de la Croix-Rouge ;

b) de reconnaître toute Société nationale de la Croix-Rouge nouvellement créé ou reconstituée et répondant aux conditions de reconnaissance en vigueur, et de notifier cette reconnaissance aux autres Sociétés nationales;

c) d'assumer les tâches qui lui sont reconnues par les Conventions de Genève, de travailler à l'application fidèle de ces Conventions et de recevoir toute plainte au sujet de violations alléguées des Conventions humanitaires ;

d) d'agir, en sa qualité d'institution neutre, spécialement en cas de guerre, de guerre civile ou de troubles intérieurs; de s'employer en tout temps à ce que les victimes militaires et civiles desdits conflits et de leurs suites directes reçoivent protection et assistance, et de servir, sur le plan humanitaire, d'intermédiaire entre les parties;

e) de contribuer, en vue desdits conflits, à la préparation et au développement du personnel et du matériel sanitaires, en collaboration avec les organisations de la Croix-Rouge et les Services de santé militaires et autres autorités compétentes;

f) de travailler au perfectionnement du droit international humanitaire, à la compréhension et la diffusion des Conventions de Genève et d'en préparer les développements éventuels;

g) d'assumer les mandats qui lui sont confiés par les Conférences internationales de la Croix-Rouge.

Le CICR peut en outre prendre toute initiative humanitaire qui entre dans son rôle d'institution spécifiquement neutre et indépendante et étudier toute question dont l'examen par une telle institution s'impose.

ART. 6 (alinéa premier). - Le CICR se recrute par cooptation parmi les citoyens suisses. Le nombre de ses membres ne peut dépasser vingt-cinq.

I La Croix-Rouge internationale comprend les Societés nationales de la Croix-Rouge, le Comité international de la Croix-Rouge et la Ligue des Sociétés de la Croix-Rouge. L'expression * Sociétéa nationales do la Croix-Rouge " couvre egalement les Sociétés du Croissant-Rouge et la Societs du Lion-et-Soleil-Rouge. 


\section{ADRESSES DES COMITÉS CENTRAUX}

AFGHANISTAN - Croissant-Rouge afghan, Kaboul.

AFRIQUE DU SUD (République) - Croix-Rouge sud-africaine, Cor. Kruis \& Market Streets, P.O.B. 8726, Johannesburg.

ALBANIE - Croix-Rouge albanaise, 35, Rruga e Barrikadavet, Tirana.

ALGERIE - Comité central du Croissant-Rouge algérien, 15 bis, boulevard Mohamed V, Alger.

ALIEMAGNE (République démocratique) - CroixRouge allemande dans la République démocratique allemande, 2, Kaitzerstrasse, Dresde $A 1$.

ALLEMAGNE (République fédérale) - CroixRouge allemande dans la République fédérale d'Allemagne, 71, Friedrich-Ebert-Allee, 5300 Bonn 1, Postfach.

ARABIE SÉOUDITE - Croissant-Rouge de l'Arabie Séoudite, Riyad.

RÉPUBLIQUE ARABE UNIE - Croissant-Rouge de la République Arabe Unie, rue Ramsès, 34, Le Caire.

ARGENTINE - Croix-Rouge argentine, H. Yrigoyen 2068, Buenos Aires.

AUSTRALIE - Croix-Rouge australienne, 122-128 Flinders Street, Melbourne, C.I.

AUTRICHE - Croix-Rouge autrichienne, 3, Gusshausstrasse, Postfach 39, Vienne $I V$.

BELGIQUE - Croix-Rouge de Belgique, 98, chaussée de Vleurgat, Bruxelles 5.

BIRMANIE - Croix-Rouge de Birmanie 42, Strand Road, Red Cross Building, Rangoon.

BOLIVIE - Croix-Rouge bolivienne, avenue SimonBolivar, 1515 (Casilla 741), La Paz.

BRÉSIL - Croix-Rouge brésilienne, Prasa da Cruz Vermelha, 10-12, Caixa postal $1286 \mathrm{zc} / 00$, Rio de Janeiro.

BULGARIE - Croix-Rouge bulgare, 1, boul. S. S. Biruzov, Sofia.

BURUNDI - Croix-Rouge du Burundi, B.P. 324 rue du Marché 3, Bujumbura.

CAMBODGE - Croix-Rouge cambodgienne, $17 \mathrm{R}$ Vithei Croix-Rouge cambodgienne, B.P. 94, Phnom-Penh.

CAMEROUN - Comité central de la Croix-Rouge camerounaise, rue Henri-Dunant, Boite postale 631, Yaoundé.

CANADA - Croix-Rouge canadienne, 95, Wellesley Street East, Toronto, 5.

CEYLAN - Croix-Rouge de Ceylan, 106, Dharmapala Mawatte, Colombo VII.

CHILI - Croix-Rouge chilienne, Avenida Santa Maria, 0150 Correo, 15, Casilla 246. V., Santiago de Chile.

CHINE - Croix-Rouge chinoise, 22, Kanmien Hutung, Pékin, E.

COLOMBIE - Croix-Rouge colombienne, Carrera 7a, No. 34-65, Apartado Nacional 1110, Bogota D.E.

CONGO - Croix-Rouge du Congo, 41, av. Valcke, B.P. 1712, Kinshasa.
CORÉE (République de) - Croix-Rouge de la République de Corée, 32-3 Ka Nam San Dong, Séoul.

CORÉE (République démocratique populaire de) Croix-Rouge de la République démocratique populaire de Corée, Pyongyang.

COSTA RICA - Croix-Rouge costaricienne, Apartado 1025, San José.

COTE D'IVOIRE Croix-Rouge de Côte d'Ivoire, B.P. 1244, Abidjan.

CUBA - Croix-Rouge cubaine, Ignacio Agramonte, 461, La Havane.

DAHOMEY - Société nationale de la Croix-Rouge du Dahomey, B.P. 1, Porto-Novo.

DANEMARK - Croix-Rouge danoise, Ny Vestergade 17 , Copenhague $K$.

RÉPUBLIQUE DOMINICAINE - Croix-Rouge dominicaine, Calle Galvan, 24, Apartado 1293, Saint-Domingue.

ÉQUATEUR - Croix-Rouge equatorienne, Calle de la Cruz Roja y avenida Colombia 118, Quito.

ESPAGNE - Croix-Rouge espagnole, Eduardo Dato, 16, Madrid, 10 .

ÉTATS-UNIS Croix-Rouge américaine, National Headquarters, 17 th and D. Streets, N.W., Washington 6, D.C.

ÉTHIOPIE - Croix-Rouge éthiopienne, Red Cross Road No 1, P.O. Box 195, Addis-Abéba.

FINLANDE - Croix-Rouge de Finlande, Tehtaankatu, 1 A, P.O.B. 14168, Helsinki 14.

FRANCE - Croix-Rouge française, 17, rue QuentinBauchart, Paris (8).

GHANA - Croix-Rouge du Ghana, P.O. Box 835, Accra.

GRANDE-BRETAGNE - Croix-Rouge britannique, 9, Grosvenor Crescent, Londres, S.W.1.

GRECE - Croix-Rouge hellénique, Rue Lycavittou, 1 , Athenes 135.

GUATÉMALA - Croix-Rouge du Guatémala, $3^{a}$ Calle 8-40, Zona 1, Guatémala C.A.

GUYANE - Croix-Rouge de Guyane, P.O. Box 351, Eve Leary, Georgetown.

HAI̋TI - Croix-Rouge hailtienne, place des NationsUnies, B.P. 1337, Port-au-Prince.

HAUTE-VOLTA - Croix-Rouge voltaïque, B.P. 340, Ouagadougou.

HONDURAS - Croix-Rouge de Honduras, Calle Henri-Dunant No 516, Tegucigalpa.

HONGRIE - Croix-Rouge hongroise, Arany Janos utca, 31, Budapest $V$.

INDE - Croix-Rouge de l'Inde, Red Cross Road 1, La Nouvelle-Dehli, 1 .

INDONÉSIE - Croix-Rouge indonésienne, Tanah Abang Barat, 66, P.O. Box 2009, Djakarta.

IRAK - Croissant-Rouge de I'Irak, Al Mansour, Bagdad.

IRAN - Société du Lion-et-Soleil-Rouge de l'Iran, Avenue Ark, Téhéran. 


\section{ADRESSES DES COMITÉS CENTRAUX}

IRLANDE - Croix-Rouge irlandaise, 16, Merrion Square, Dublin 2.

ISLANDE - Croix-Rouge islandaise, øldugøtu 4, Post Box 872. Reykjavik.

ITALIE - Croix-Rouge italienne, 12, via Toscana, Rome.

JAMAIQUE - Croix-Rouge de la Jamaique, 76, Arnold Road, Kingston 5.

JAPON - Croix-Rouge du Japon, 5, Shiba Park, Minato-Ku, Tokio.

JORDANIE - Croissant-Rouge jordanien, P.O. Box 10001, Amman.

KÉNYA - Croix-Rouge du Kénya, St. John's Gate, B.O. Box 712, Nairobi.

KOWEIT - Croissant-Rouge de Koweï, P,O. Box 1359, Koweit.

LAOS - Croix-Rouge lao, B.P. 650, Vientiane.

LIBAN - Croix-Rouge libanaise, rue GénéralSpears, Beyrouth.

LIBÉRIA - Croix-Rouge đu Libéria, National Head quaters, Corner of Tubman boulevard and $9 \mathrm{~h}$ Street Sinkor, P.O. Box 226, Monrovia.

LIBYE - Croissant-Rouge libyen, Berka Omar Mukhtar Street, P.O. Box 541, Benghazi.

LIECHTENSTEIN - Croix-Rouge du Liechtenstein, Vaduz.

LUXEMBOURG - Croix-Rouge luxembourgeoise, Parc de la Ville, C.P. 234, Luxembourg.

MADAGASCAR - Croix-Rouge de la République malgache, rue Clemenceau, B.P. 1168, Tananarive.

MALAISIE - Croix-Rouge de Malaisie, Jalan Belfield 519, Kuala Lumpur.

MALI - Croix-Rouge malienne, Route de Koulikora, B.P. 280, Bamako.

MAROC - Croissant-Rouge marocain, rue Benzakour, B.P. 189, Rabat.

MEXIQUE - Croix-Rouge mexicaine, Avenida Ejercito Nacional No 1032, Mexico, 10, DF.

MONACO - Croix-Rouge monégasque, bd de Suisse, 27, Monte-Carlo.

MONGOLIE (République populaire de) - CroixRouge de la République populaire de Mongolie, Central Post Office, Post Box 537, Oulan-Bator.

NÉPAL - Croix-Rouge du Népal, Tripureswore, P.B. 217, Kathmandu.

NICARAGUA - Croix-Rouge du Nicaragua, 12, Avenida Noroeste, Managua, D.N.

NIGER - Croix-Rouge du Niger, B.P. 386, Niamey.

NIGERIA - Croix-Rouge du Nigéria, Eko Akete Close, Ikoyi, Yaba, P.O. Box 764, Lagos.

NORVĖGE - Croix-Rouge de Norvège, Parkveien, 33b, Oslo 3.

NOUVELLE-ZÉLANDE - Croix-Rouge néo-zélandaise, 61, Dixon Street, P.O.B. 6073, Wellington, C.2.

OUGANDA - Croix-Rouge de l'Ouganda, 57, Roseberry Street, P.O. Box 494, Kampala.

PAKISTAN - Croix-Rouge du Pakistan, Frere Street, Karachi 4.

PANAMA - Croix-Rouge de Panama, Apartado 668, Panama.

PARAGUAY - Croix-Rouge paraguayenne, calle Andre Barbero y Artigas 33, Asuncion.
PAYS-BAS - Croix-Rouge néerlandaise, 27, Prinsessegracht, La Haye.

PÉROU - Croix-Rouge péruvienne, Jiron Chancay 881, Lima.

PHILIPPINES - Croix-Rouge philippine, 860 , United Nations Avenue, P.O. Box 280, Manille.

POLOGNE - Croix-Rouge polonaise, Mokotowska 14, Varsovie.

PORTUGAL - Croix-Rouge portugaise, Secrétariat général, Jardim 9 de Abril, 1-5, Lisbonne 3.

ROUMANIE - Croix-Rouge de la République Socialiste de Roumanie, Strada Biserica Amzei, 29, Bucarest.

SAINT-MARIN - Croix-Rouge de Saint-Marin, Palais gouvernemental, Saint-Marin.

SALVADOR, EL - Croix-Rouge du Salvador, $3^{a}$ avenida Norte y $3^{a}$, calle Poniente, 21, San Salvador.

SÉNÉGAL - Croix-Rouge sénégalaise, Bld. Franklin-Roosevelt, P.O.B. 299, Dakar.

SIERRA LEONE - Croix-Rouge de Sierra Leone, P.O. Box 427, 6, Liverpool Street, Freetown.

SOUDAN - Croissant-Rouge soudanais, P.O. Box 235, Khartoum.

SUÈDE - Croix-Rouge suédoise, Artillerigatan, 6, 10440 Stockholm 14.

SUISSE - Croix-Rouge suisse, Taubenstrasse, 8, Case postale 2699, 3001 Berne.

SYRIE - Croissant-Rouge syrien, 13, rue Abi-AlaAlmaari, Damas.

TANZANIE - Croix-Rouge de Tanganyika, Upanga Road, P.O.B. 1133, Dar-es-Salaam.

TCHÉCOSLOVAQUIE - Croix-Rouge tchécoslovaque, Thunovska, 18, Prague $I$.

THAIlLANDE - Croix-Rouge thallandaise, King Chulalongkorn Memorial Hospital, Bangkok.

TOGO - Croix-Rouge togolaise, 19, avenue des Alliés, B.P. 655, Lomé.

TRINITÉ-et-TOBAGO - Croix-Rouge de Triniteet-Tobago, 48, Pembroke Street, P.O. Box 357, Port of Spain.

TUNISIE - Croissant-Rouge tunisien, 19, rue d'Angleterre, Tunis.

TURQUIE - Société du Croissant-Rouge turc, Yenisehir, Ankara.

U.R.S.S. - Alliance des Sociétés de la Croix-Rouge et du Croissant-Rouge de ]'U.R.S.S., Tchoremushkinskii proezd 5, Moscou, $W 36$.

URUGUAY - Croix-Rouge uruguayenne, Avenida 8 de Octubre 2990, Montevideo.

VENEZUELA - Croix-Rouge vénézuélienne, Avenida de Andrés Bello, No. 4, Apart. 3185, Caracas.

VIETNAM (République démocratique du) - CroixRouge de la République démocratique du Vietnam, 68, rue Bd-Triè, Hanol.

VIETNAM (République du) - Croix-Rouge de la République du Vietnam, rue Hông Thâp Tu, No 201, Saigon.

YOUGOSLAVIE - Croix-Rouge yougoslave, Simina ulica broj, 19, Belgrade.

ZAMBIE - Croix-Rouge de Zambie, P.O. Box R. W. 1, Lusaka.

Imprimerie du Journal de Genève, Genève. 